\title{
The effects of exercise on portal venous system in splenectomized adults
}

\author{
Ozban $\mathrm{M}^{1}$, Genc $\mathrm{V}^{1}$, Karaca $\mathrm{S}^{1}$, Cetinkaya $\mathrm{OA}^{1}$, Oztuna $\mathrm{D}^{2}$ \\ Department of Surgery, Ankara University School of Medicine, Ankara, Turkey. \\ volkan@medicine.ankara.edu.tr
}

\begin{abstract}
Objectives: This study was designed to assess the effects of exercise on the portal venous system in splenectomized adults.

Background: Splenectomy is a surgical intervention commonly performed at surgery clinics and recognized as a cause of portal vein thrombosis. Intensive exercise increases blood flow to the contracting muscles, causes hypercoagulability and vasoconstriction in the splanchnic area, hence the portal vein diameter, blood flow velocity and discharge decrease.

Methods: Forty adults participated in this investigation. Subjects were trained to run for twenty minutes on a treadmill at a velocity of $6 \mathrm{~km} / \mathrm{h}$ and ten-degree elevation. We compared the white blood cell count, portal vein diameter, portal venous blood flow velocity and discharge of splenectomized and healthy adults before and after exercise.

Results: After exercise, the blood leukocyte count was significantly increased and the portal vein diameter was significantly reduced in both groups $(p<0.001)$ but there were no statistically significant differences between the two groups. The portal venous blood flow rate in splenectomy group were significantly lower than in the control group $(p<0.001)$. Furthermore the portal venous blood flow rates in both groups were significantly reduced after exercise $(p<0.001)$.

Conclusion: Exercise in splenectomized individuals can cause serious problems in form of decreasing splanchnic flow and increasing blood viscosity. After splenectomy, both healthy individuals and patients with hematologic diseases ought to avoid intensive exercises (Tab. 2, Ref. 16). Full Text in PDF www.elis.sk.

Key word: splenectomy, blood flow velocity, portal vein, thrombosis.
\end{abstract}

Splenectomy due to hematologic disorders or trauma is a common operation in surgery clinics. Portal vein thrombosis is a rare but fatal complication of splenectomy (1). Portal vein diameter per se does not change after splenectomy but the splenic vein, being a component of the portal venous system becomes nonfunctional and hence the portal venous blood flow decreases.

The splenectomized human population is usually young and continues leading an active life in their post-operative period. Intensive exercise increases the blood flow to the contracting muscles while causing hypercoagulability and vasoconstriction in the splanchnic area; hence the portal venous diameter, blood flow velocity and discharge decrease (2).

This study was designed to assess the effects of exercise on portal venous system in splenectomized adults. Therefore we compared the portal vein cross-sectional area, portal venous blood flow, and portal vein discharge in splenectomized and healthy adults. We also compared other parameters affected by exercise such as body temperature and white blood cell count.

${ }^{1}$ Department of Surgery, Ankara University School of Medicine, Ankara, Turkey, and ${ }^{2}$ Department of Biostatistics, Ankara University School of Medicine, Ankara, Turkey

Address for correspondence: V. Genc MD, Cigdem Mah. 28. Cad. 13/23, 06520 Cankaya Ankara Turkey.

Phone: +90.312 .3103333 , Fax: +90.312 .3103989$

\section{Methods}

The study was approved by the Faculty Ethics Committee at our institution. The methods and possible risks were explained to the subjects in detail and they gave written informed consent before participating.

\section{Subjects}

Twenty adults who underwent splenectomy for benign masses of spleen, ITP or trauma were compared with 20 healthy adults. The patients participating in the study underwent splenectomy at least six months before the study, their age ranged from 15 to 45 years, and they were suitable for exercise in cardiac and other aspects. The patients with portal vein thrombosis, myeloproliferative diseases, hemolytic and lymphoproliferative disorders were excluded from the study.

Protocol

After eight-hour fasting with only water permitted, the subjects arrived at the laboratory. White blood cell count, heart rate and body temperature of the subjects were measured before exercise. The portal vein diameter, and velocity of portal venous blood flow were measured by B\&K Medical ultrasound system Panther 2002 equipped (B\&K Medical, Gentofte, Denmark) with 3.5 MHz convex transducer. Measurements were made to the right 
of the midline subcostal or intercostal region in a supine position during breath-hold. The portal vein cross-sectional area was calculated from equation

$$
\operatorname{pvca}\left(m m^{2}\right)=(p v r)^{2} \times \pi,
$$

where pvr is portal vein radius ( $\mathrm{mm}$ ) and $\pi$ (pi) approximately equals 3.14159 (3). The portal vein discharge was calculated according to the relationship:

$$
p v d(m l / s)=(p b f) x(p v c a),
$$

where pbf is portal venous blood flow $(\mathrm{mm} / \mathrm{s})$ and pvca is described above (4). The subjects were trained to run on a treadmill for 20 minutes at a velocity of $6 \mathrm{~km} / \mathrm{h}$ and ten-degree elevation. During the exercise, the subjects were monitored for their heart rate, respiration pattern and $\mathrm{O}_{2}$ saturation. The analysis of white blood cells, heart rate, body temperature, portal vein diameter and blood flow velocity measurements were performed after the completion of exercise and subsequently the portal vein cross-sectional area and discharge were recalculated. Each subject properly completed the exercise protocol.

\section{Data analysis}

Statistical analyses were performed using SPSS 11.5 (Statistical Packages for Social Sciences). Data were expressed as mean \pm standard deviation for metric variables. In order to compare splenectomy and control groups in terms of metric variables, the two-sample $t$ test was used. For categorical variables chi-square test was used. For the within-group comparisons, the paired $t$ test was performed. $p<0.05$ was accepted as statistically significant.

\section{Results}

Demographic data, hemoglobin, hematocrit, and platelet counts per group are shown in Table 1. Both groups were anthropometrically similar and the mean ages of splenectomy and control

Tab. 1. Anthropometric characteristics and hematological data of subjects.

\begin{tabular}{lccc}
\hline & $\begin{array}{c}\text { Splenectomy } \\
(\mathrm{n}=20)\end{array}$ & $\begin{array}{c}\text { Control } \\
(\mathrm{n}=20)\end{array}$ & $\begin{array}{c}\mathrm{p} \\
\text { value }\end{array}$ \\
\hline Age(years) & $29.95 \pm 6.64$ & $30.40 \pm 6.06$ & 0.841 \\
Male sex $(\mathrm{n})$ & 16 & 14 & 0.465 \\
Height $(\mathrm{cm})$ & $167.85 \pm 6.21$ & $168.30 \pm 6.11$ & 0.883 \\
Weight(kg) & $73.45 \pm 5.70$ & $74.2 \pm 7.09$ & 0.738 \\
Hemoglobin(g/dl) & $14.69 \pm 1.10$ & $14.01 \pm 1.22$ & 0.072 \\
Hematocrit $(\%)$ & $43.10 \pm 3.57$ & $42.55 \pm 3.00$ & 0.445 \\
Platelets(x109/L) & $403.20 \pm 49.92$ & $300.75 \pm 75.83$ & $<0.001$ \\
\hline
\end{tabular}

Results are expressed as the mean \pm SD. groups were 29.9 and 30.4, respectively. Indications for splenectomy in patients included trauma $(n=9), \operatorname{ITP}(n=8)$, hemangioma $(n=1)$ and cyst $(n=2)$. Hematocrit and hemoglobin measurements were the same in both groups. The splenectomized subjects had their statistical platelet values $\left(403 \times 10^{9} / \mathrm{L}\right)$ significantly higher than the control subjects $\left(300 \times 10^{9} / \mathrm{L} ; \mathrm{p}<0.001\right)$.

There was no statistical difference between the groups in respect of body temperatures, blood leukocyte counts and portal vein diameters. But when these paired as before and after exercise and in this way compared in each group, after exercise, a statistically significant increase in body temperatures, blood leukocyte counts, and statistically significant decrease in portal vein diameter were determined $(\mathrm{p}<0.001)$.

The portal vein blood flow velocity and discharge were lower in splenectomy group than in control group $(\mathrm{p}<0.001)$. When these paired as before and after exercise and in this way compared in each group, after exercise, in both groups, statistically significant decreases were determined $(\mathrm{p}<0.001)$. Before and after exercise body temperature, white blood cell count, portal vein diameter, velocity of portal venous blood flow, and portal vein discharge values are shown in Table 2.

\section{Discussion}

The portal vein diameter does not change after splenectomy but while being a component of portal venous system, the splenic vein becomes nonfunctional and the portal venous blood flow significantly decreases. Portal vein thrombosis is a well-known fatal and life-threatening complication of splenectomy. Post-splenectomy portal vein thrombosis is about $2-11 \%(5,6)$. Splenectomy is the most important etiologic factor for portal vein thrombosis. If the reason for splenectomy is based on any of myeloproliferative diseases, hemolytic disorders or lymphoproliferative diseases, then post-splenectomy thromboses commonly occur (7). We excluded patients with all these diseases from our trial to standardize the splenectomized adults.

During exercise, the main part of blood is "stolen" from the gastrointestinal system; therefore the effect of exercise on gastrointestinal system has been commonly investigated. Splanchnic blood is redistributed to peripheral muscles in accord with exercise intensity. One of the main factors determining the splanchnic hemodynamics is the portal blood flow and it can be used to analyze the splanchnic blood flow $(2,8)$.

The portal venous blood flow used to be measured by indocyanin green clearance and its significant decrease could be determined albeit only after a period of severe exercise (9). Today with the development of technology, the portal venous blood flow and

Tab. 2. Before and after exercise values of body temperature, white blood cell, portal vein diameter, portal vein blood velocity and portal vein blood flow.

\begin{tabular}{lllll}
\hline & BE splenectomy & AE splenectomy & BE control & AE control \\
\hline Body temperature & $36.45 \pm 0.29$ & $37.63 \pm 0.35$ & $36.51 \pm 0.18$ & $37.84 \pm 0.21$ \\
WBC $(/ \mathrm{mm} 3)$ & $7720 \pm 1582$ & $10653 \pm 1646$ & $7555 \pm 1778$ & $10480 \pm 1703$ \\
Portal vein diameter $(\mathrm{mm})$ & $9.47 \pm 1.28$ & $7.62 \pm 1.35$ & $9.67 \pm 0.83$ & $7.72 \pm 0.96$ \\
Portal vein blood velocity $(\mathrm{mm} / \mathrm{s})$ & $118.51 \pm 15.10$ & $82.99 \pm 13.03$ & $348.49 \pm 43.92$ & $177.43 \pm 32.99$ \\
Portal vein blood flow $(\mathrm{ml} / \mathrm{s})$ & $8.55 \pm 2.74$ & $3.86 \pm 1.40$ & $27.12 \pm 8.59$ & $\mathrm{p}<0.001 \mathrm{a}$ \\
\hline
\end{tabular}

BE: before exercise, AE: after exercise. Data are expressed as the mean \pm SD. a Statistically significant difference was found only compared values of before and after-exercise in each group.b Statistically significant difference was found not only compared values of before and after-exercise in each group but also compared between splenectomy and control group. 
thrombosis can easily be determined by contrast-enhanced computerized tomography (CT) and ultrasonography (USG). Bach et al. showed that colored Doppler USG has a specificity of $99 \%$ and sensitivity of $93 \%$ while CT has a specificity of $99 \%$ and sensitivity of $90 \%$ (10). We preferred colored Doppler USG because of its speed and easy application for examining the portal vein system.

The exercise was carried out under laboratory conditions; the subjects were trained to run for 20 minutes at a velocity of $6 \mathrm{~km} / \mathrm{h}$. Rehrer et al (2) used a severe sixty-minute exercise program for healthy individuals but we did not use the same program with our splenectomized subjects in order to prevent the portal vein blood flow from undue excessive decreasing.

There are many studies related to portal blood flow and exercise. Brown et al. analyzed the effect of postural position on portal vein blood flow. They determined a $26 \%$ decrease in blood flow in standing position compared with supine position (11). We did not analyze the same situation in our trial but all measurements were done in supine position for standardization. Rehrer et al. investigated the effect of exercise on portal venous blood flow in healthy men while the portal vein diameter, cross-sectional area and blood flow rates were found significantly decreased after exercise (2). A study done by Rao et al.found the gastrointestinal symptoms to increase in individuals who had regular sportive activity, especially marathon runners. They suggested some possible reasons including the decrease in splanchnic blood flow, changes in the levels of prostaglandins and other gastrointestinal hormones that could be affected by exercise (12). We also determined that the velocity of portal venous blood flow and portal vein discharge decreased after exercise in both splenectomized and healthy subjects. At the same time we showed that rather than the control group, the splenectomized group showed a significant decrease in portal blood flow rates.

It is clear that exercise does not alter only the portal blood flow, but it changes also the quantity and quality of the immune system cells with tissue destruction and production of stress hormones. As opposed to regular and mild exercises which decrease the infection rates when compared to sedentary life style, severe exercises cause immunologic disorders (13). Natela et al showed that severe exercise caused tissue destruction; therefore stress hormones were secreted thus altering the levels of various immunologic cells and functions (14). Depending especially on their intensity and duration, acute exercises increase the leukocyte counts (particularly those of lymphocytes and neutrophils), raise many inflammatory cytokines affecting the functions of leukocytes (TNF-alfa, IL-1beta), and increase anti-inflammatory cytokines (IL-6, IL-10) and acute phase reactants (CRP) (13). Timmons et al. analyzed the effect of age and sex on the levels of leukocytes after exercise. The latter factors were found to increase the leukocyte counts after exercise in all groups but especially significantly in females (15). We did not identify any differences between splenectomized and healthy groups in respect to levels of leukocyte but we showed a statistically significant increase in leukocyte counts after exercise. It is known that blood viscosity may cause thrombosis and thromboembolic complications. Ho et al. showed that blood components could affect the whole blood viscosity (16). Therefore leukocytosis especially in splenectomized adults may cause thrombosis resulting in portal vein thrombosis.

\section{Conclusion}

During exercise, the rate of blood flow to the muscles and blood viscosity increase, while the splanchnic area reacts by vasoconstriction. All these factors cause a decrease in portal vein diameter, blood flow and discharge. Splenectomy may cause portal vein thrombosis. Exercise in splenectomized individuals can cause serious problems such as decrease in splanchnic blood flow and increase in blood viscosity. After splenectomy, both healthy individuals and patients with hematologic diseases ought to avoid intensive exercises.

\section{References}

1. Rattner DW, Ellman L, Warshaw AL. Portal vein thrombosis after elective splenectomy: an underappreciated, potentially lethal syndrome. Arch Surg 1993; $128: 565$.

2. Rehrer NJ, Smets A, Reynaert H, Goes E, De Meirleir K. Effect of exercise on portal vein blood flow in man. Med Sci Sports Exerc 2001; 33 (9): 1533-1537.

3. Edwards CH, Penney DE. Calculus with analytic geometry. 4th ed. PracticeHall international edition: A Simon\& Schuster company; 1994, 5 p.

4. French RH. Open-channel hydraulics. 2nd ed. B \& JO Enterprice PTE LTD: McGraw-Hill Book Company; 1987, 22 p.

5. Petit P, Bret PM, Atri M, Hreno A, Casola G, Gianfelice D. Splenic vein thrombosis after splenectomy: frequency and role of imaging. Radiology 1994; 190 (1): 65-68.

6. van't Riet M, Burger JW, van Muiswinkel JM, Kazemier G, Schipperus MR, Bonjer HJ. Diagnosis and treatment of portal vein thrombosis following splenectomy. Br J Surg 2000; 87 (9): 1229-1233.

7. Romano F, Caprotti R, Conti M et al. Thrombosis of the splenoportal axis after splenectomy. Langenbecks Arch Surg 2006; 391 (5): 483-488.

8. Zoli M, Marchesini G, Cordiani MR et al. Echo-Doppler measurement of splanchnic blood flow in control and cirrhotic subjects. J Clin Ultrasound 1986; 14 (6): 429-435.

9. Rowell LB, Blackmon JR, Bruce RA. Indocyanine gren clearance and estimated blood flow during mild to maximal exercise in upright man. J Clin Invest 1964; 43: 1677-1690.

10. Bach AM, Hann LE, Brown KT et al. Portal vein evaluation with US: comparison to angiography combined with CT arterial portography. Radiology 1996; 201: 149-154.

11. Brown HS, Halliwell M, Qamar M, Read AE, Evans JM, Wells PN. Measurement of normal portal venous blood flow by Doppler ultrasound. Gut 1989; 30 (4): 503-509.

12. Rao KA, Yazaki E, Evans DF, Carbon R. Objective evaluation of small bowel and colonic transit time using $\mathrm{pH}$ telemetry in athletes with gastrointestinal symptoms. Br J Sports Med 2004; 38 (4): 482-487.

13. Gleeson M. Immune function in sport and exercise. J Appl Physiol 2007; 103: 693-699.

14. Natela VM, Brenner IK, Moldoveanu AI, Vasiliou P, Shek P, Shephard RJ. Effects of three different types of exercise on blood leukocyte count during and following exercise. Sao Paulo Med J 2003; 121 (1): 9-14.

15. Timmons BW, Tarnopolsky MA, Sinder DP, Bar-Or O. Immunological changes in response to exercise: influence of age, puberty, and gender. Med Sci Sports Exerc 2006; 38 (2): 293-304.

16. Ho CH. White blood cell and platelet counts could affect whole blood viscosity. J Chin Med Assoc 2004; 67 (8): 394-397.

Received March 4, 2010. Accepted February 20, 2012. 